\title{
Oscillation of Two-Dimensional Neutral Delay Dynamic Systems
}

\author{
Xinli Zhang and Shanliang Zhu \\ College of Mathematics and Physics, Qingdao University of Science and Technology, Qingdao 266061, China
}

Correspondence should be addressed to Xinli Zhang; zhangxinli1000@yahoo.com.cn

Received 15 January 2013; Accepted 12 March 2013

Academic Editor: Changpin Li

Copyright (C) $2013 \mathrm{X}$. Zhang and S. Zhu. This is an open access article distributed under the Creative Commons Attribution License, which permits unrestricted use, distribution, and reproduction in any medium, provided the original work is properly cited.

We consider a class of nonlinear two-dimensional dynamic systems of the neutral type $\left(x(t)-a(t) x\left(\tau_{1}(t)\right)\right)^{\Delta}=p(t) f_{1}(y(t)), y^{\Delta}(t)=$ $-q(t) f_{2}\left(x\left(\tau_{2}(t)\right)\right)$. We obtain sufficient conditions for all solutions of the system to be oscillatory. Our oscillation results when $a(t)=0$ improve the oscillation results for dynamic systems on time scales that have been established by Fu and Lin (2010), since our results do not restrict to the case where $f(u)=u$. Also, as a special case when $\mathbb{T}=\mathbb{R}$, our results do not require $a_{n}$ to be a positive real sequence. Some examples are given to illustrate the main results.

\section{Introduction}

In this paper, we are concerned with oscillation of the twodimensional nonlinear neutral dynamic systems

$$
\begin{aligned}
& \left(x(t)-a(t) x\left(\tau_{1}(t)\right)\right)^{\Delta}=p(t) f_{1}(y(t)), \\
& y^{\Delta}(t)=-q(t) f_{2}\left(x\left(\tau_{2}(t)\right)\right),
\end{aligned}
$$

on time scales. Since we are interested in the oscillatory behavior of the solution of system (1) near infinity, we will assume throughout this paper that the time scales $\mathbb{T}$ are unbounded. We assume that $t_{0} \in \mathbb{T}$, and it is convenient to let $t_{0}>0$, and define the time scale interval $t \in\left[t_{0}, \infty\right)_{\mathbb{T}}$ by $t \in\left[t_{0}, \infty\right)_{\mathbb{T}}:=\left[t_{0}, \infty\right) \cap \mathbb{T}$. For system (1), we assume that

$\left(H_{1}\right) a(t) \in C\left(\left[t_{0}, \infty\right)_{\mathbb{T}}, \mathbb{R}\right)$, and $-1<a(t) \leq 1 ;$

$\left(H_{2}\right) \tau_{i}(t)=t-\delta_{i}$, where $\delta_{i} \geq 0, i=1,2 ;$

$\left(H_{3}\right) p(t)$ and $q(t)$ are real valued positive and rdcontinuous functions defined on $\mathbb{T}$, and $\int_{t_{0}}^{\infty} p(t) \Delta t=$ $\infty$;

$\left(H_{4}\right) f_{i}: \mathbb{R} \rightarrow \mathbb{R}$ are continuous, nondecreasing with $u f_{i}(u)>0$ for $u \neq 0, i=1,2$. There exists continuous function $h: \mathbb{R} \times \mathbb{R} \rightarrow \mathbb{R}$ such that $f_{1}(u)-f_{1}(v)=h(u, v)(u-v)$ for all $u \neq v$, and $h(u, v)>$ $\beta_{1}>0$ for all $u, v \in \mathbb{R} .\left|f_{2}(u)\right| \geq \beta_{2}|u|$, where $\beta_{2}$ is a positive constant.
The theory of time scales, which has recently a lot of attention, was introduced by Hilger in his Ph.D. degree thesis in 1988 in order to unify continuous and discrete analysis (see [1]). Not only can this theory of the so-called "dynamic equations" unify the theories of differential equations and difference equations, but also extend these classical cases to cases "in between," for example, to the so-called $q$-difference equations and can be applied on other different types of time scales. Since Hilger formed the definition of derivatives and integrals on time scales, several authors have expounded on various aspects of the new theory; see the paper in [2] and the references cited therein. A book on the subject of time scales in [3] summarizes and organizes much of time scale calculus. The reader is referred to [3], Chapter 1, for the necessary time scale definitions and notations used throughout this paper.

Our main interest in this paper is to establish some oscillation results for system (1). We will relate our results to some earlier work for system (1). In the special case when $\mathbb{T}=\mathbb{N}$, system (1) becomes the two-dimensional difference system

$$
\begin{aligned}
& \Delta\left(x_{n}-a_{n} x_{\tau_{1}(n)}\right)=p_{n} f_{1}\left(y_{n}\right), \\
& \Delta y_{n}=-q_{n} f_{2}\left(x_{\tau_{2}(n)}\right) .
\end{aligned}
$$

If $a_{n}$ is a positive real sequence, the oscillatory property of system (2) has been receiving attention. We refer the reader to the papers $[4,5]$ and the references cited therein. However, 
system (1) have been restricted to the case when $0<a(t) \leq 1$ in paper [4].

On the other hand, system (1) reduces to some important second-order dynamic equations in the particular case; for example,

$$
\begin{gathered}
\left(r(t) x^{\Delta}(t)\right)^{\Delta}+p(t) f(x(\tau(t)))=0, \\
\left(p(t)\left([y(t)+r(t) y(\tau(t))]^{\Delta}\right)^{\gamma}\right)^{\Delta}+f(t, y(\theta(t)))=0,
\end{gathered}
$$$$
\gamma \geq 1
$$

We refer the reader to the recent papers [6-9] and the references cited therein. However, there are few works about oscillation of dynamic systems on time scales, motivated by [4] and the references cited therein, and in this paper, we investigate oscillatory properties for system (1). In Section 2, we present some basic definitions concerning the calculus on time scales. In Section 3, we discuss the case $0<a(t) \leq 1$; the case $-1<a(t) \leq 0$ will be studied in Section 4 . Examples are given in Section 5 to illustrate our theorems.

\section{Preliminary}

For completeness, we recall the following concepts and results concerning time scales that we will use in the sequel. More details can be found in [10-12].

The forward and backward jump operators are defined by

$$
\sigma(t):=\inf \{s \in \mathbb{T}: s>t\}, \quad \rho(t):=\sup \{s \in \mathbb{T}: s<t\},
$$

where $\inf \emptyset:=\sup \mathbb{T}$ and $\sup \emptyset:=\inf \mathbb{T}$, where $\emptyset$ denotes the empty set. A point $t \in \mathbb{T}$ is called left-dense if $t>\inf \mathbb{T}$ and $\rho(t)=t$, right-dense if $t<\sup \mathbb{T}$ and $\sigma(t)=t$, leftscattered if $\rho(t)<t$, and right-scattered if $\sigma(t)>t$. A function $g: \mathbb{T} \rightarrow \mathbb{R}$ is said to be rd-continuous if it is continuous at every right-dense point and if the left-sided limit exists at every left-dense point. The set of all such rd-continuous functions is denoted by $C_{\mathrm{rd}}(\mathbb{T})$. The graininess function $\mu$ for a time scale $\mathbb{T}$ is defined by $\mu(t):=\sigma(t)-t$, and for any function $f(t): \mathbb{T} \rightarrow \mathbb{R}$, the notation $f^{\sigma}(t)$ denotes $f(\sigma(t))$.

A function $P: \mathbb{T} \rightarrow \mathbb{R}$ is called positively regressive (we write $p \in \Re^{+}$) if it is rd-continuous function and satisfies $1+\mu(t) p(t)>0$ for all $t \in \mathbb{T}$. For a function $f: \mathbb{T} \rightarrow \mathbb{R}$, the (delta) derivative is defined by

$$
f^{\Delta}(t)=\frac{f(\sigma(t))-f(t)}{\sigma(t)-t},
$$

if $f$ is continuous at $t$ and $t$ is right-scattered. If $t$ is not rightscattered, then the derivative is defined by

$$
f^{\Delta}(t)=\lim _{s \rightarrow t} \frac{f(t)-f(s)}{t-s},
$$

provided this limit exists. A function $f:[a, b] \rightarrow \mathbb{R}$ is said to be right-dense continuous if it is right continuous at each right-dense point and there exists a finite left limit at all leftdense points, and $f$ is said to be differentiable if its derivative exists. A useful formula is

$$
f^{\sigma}=f(\sigma(t))=f(t)+\mu(t) f^{\Delta}(t) .
$$

Assume that $f, g: \mathbb{T} \rightarrow \mathbb{R}$ are differentiable at $t \in \mathbb{T}$ and $f(t) f^{\sigma}(t) \neq 0$; then, $g / f$ is differentiable at $t$ and

$$
\left(\frac{g}{f}\right)^{\Delta}(t)=\frac{f(t) g^{\Delta}(t)-f^{\Delta}(t) g^{\sigma}(t)}{f(t) f^{\sigma}(t)} .
$$

If $f, g \in C_{\mathrm{rd}}$ and $a, b \in \mathbb{T}$, then

$$
\int_{a}^{b} f(t) g^{\Delta}(t) \Delta t=(f g)(b)-(f g)(a)-\int_{a}^{b} f^{\Delta}(t) g^{\sigma}(t) \Delta t .
$$

Assume that $g: \mathbb{T} \rightarrow \mathbb{R}$ is continuously differentiable and $f: \mathbb{T} \rightarrow \mathbb{R}$ is delta differentiable. Then, $g \circ f: \mathbb{T} \rightarrow \mathbb{R}$ is differentiable and

$$
(g \circ f)^{\Delta}(t)=\int_{0}^{1} g^{\prime}\left(f(t)+h \mu(t) f^{\Delta}(t)\right) d h f^{\Delta}(t) .
$$

Hilger [1] showed that for $p(t)$ to be rd-continuous and regressive, the solution of the initial value problem

$$
y^{\Delta}+p(t) y=0, \quad y\left(t_{0}\right)=1
$$

is given by

$$
y(t)=\exp \left\{\int_{t_{0}}^{t} \zeta_{\mu(s)}(-p(s)) \Delta s\right\} y\left(t_{0}\right)=e_{-p}\left(t, t_{0}\right) y\left(t_{0}\right),
$$

where

$$
\begin{gathered}
\zeta_{h}(z)= \begin{cases}\frac{\log (1+h z)}{h}, & h \neq 0, \\
z, & h=0,\end{cases} \\
e_{p}(t, s)=\exp \left\{\int_{s}^{t} \zeta_{\mu(\tau)}(p(\tau)) \Delta \tau\right\}, \quad s, t \in \mathbb{T} .
\end{gathered}
$$

\section{The Case $0<a(t) \leq 1$}

In this section, we always assume that

$$
0<a(t) \leq 1 .
$$

For any $x(t)$, we define $z(t)$ by

$$
z(t)=x(t)-a(t) x\left(\tau_{1}(t)\right) .
$$

In the following, we will give some lemmas which are important in proving our first results.

Lemma 1. Suppose that $\left(\mathrm{H}_{2}\right)-\left(\mathrm{H}_{4}\right)$ and (14) hold, and $(x(t), y(t))$ is a solution of system (1) with $x(t)$ eventually of one sign for $t \in\left[t_{0}, \infty\right)_{\mathbb{T}}$. Then, $(x(t), y(t))$ is nonoscillatory, and there exists $t_{1} \in\left[t_{0}, \infty\right)_{\mathbb{T}}$ such that $z(t)$ and $y(t)$ are monotone for $t \in\left[t_{1}, \infty\right)_{\mathbb{T}}$. 
Proof. Assume that $(x(t), y(t))$ is a solution of system (1) and $x(t)$ is nonoscillatory. Then, in view of $\left(H_{3}\right)$ and the hypothesis on $f_{2}$, from the second equation of system (1), we have either $y^{\Delta}(t) \leq 0$ or $\geq 0$ for all $t \geq t_{1} \geq t_{0}$. Thus $y(t)$ is monotone and $y(t)$ is eventually of one sign for all sufficiently large $t \geq t_{2}$. Now, from the first equation of system (1), we can prove that $z(t)$ is monotone and nonoscillatory for all sufficiently large $t \geq t_{2}$. This completes the proof of the lemma.

Lemma 2. Suppose that $\left(\mathrm{H}_{2}\right)$ and (14) hold. Let $x(t)$ be a nonoscillatory solution of the inequality

$$
x(t)\left[x(t)-a(t) x\left(\tau_{1}(t)\right)\right] \leq 0
$$

defined for all sufficiently large $t$. Then, $x(t)$ is bounded.

Proof. Without loss of generality, we may assume that $x(t)$ is an eventually positive solution of inequality (16), and the proof for the case $x(t)$ eventually negative is similar. From (16), we have

$$
x(t)-a(t) x\left(\tau_{1}(t)\right) \leq 0
$$

for all sufficiently large $t$. In view of (14), we have

$$
x(t) \leq a(t) x\left(\tau_{1}(t)\right) \leq x\left(\tau_{1}(t)\right) .
$$

Hence, $x(t)$ is bounded.

We now establish some sufficient conditions for the oscillation of (1) by reducing our study to a first-order delay dynamic inequality where we apply the results of Zhang and Deng [12]. The main result from [12] is the following lemma.

Lemma 3. Assume that $b(t)>0, \tau(t)<t$ and $\lim _{t \rightarrow \infty} \tau(t)=$ $\infty$. If

$$
\lim _{t \rightarrow \infty} \sup \sup _{\lambda>0,-\lambda b \in \mathfrak{R}^{+}} \lambda e_{-\lambda b}(t, \tau(t))<1,
$$

then the inequality

$$
x^{\Delta}(t)+b(t) x(\tau(t)) \leq 0
$$

cannot have an eventually positive solution, and the inequality

$$
x^{\Delta}(t)+b(t) x(\tau(t)) \geq 0
$$

cannot have an eventually negative solution.

Now, we state and prove our main theorem.

Theorem 4. Assume that $a(t)$ is bounded and $f_{1} \in C^{1}(\mathbb{R}, \mathbb{R})$ with $f_{1}^{\prime}(u) \geq K>0$. Denote that $A(t)=\int_{t_{0}}^{t} p(s) \Delta s$. If there exists constant $k$ such that $\delta_{2}>k+\delta_{1}$ such that

$$
\begin{aligned}
& \lim _{t \rightarrow \infty} \sup \left\{A(t) \int_{t+\delta_{2}}^{\infty} q(s) \Delta s\right\}>\frac{1}{\beta_{1} \beta_{2}}, \\
& \lim _{t \rightarrow \infty} \sup \sup _{\lambda>0,-\lambda p \in \Re^{+}} \\
& \times \lambda e_{-\lambda \beta_{1} \beta_{2} p \int_{s}^{s+k}\left(q(u) / a\left(u-\delta_{2}+\delta_{1}\right)\right) \Delta u} \\
& \times\left(t, t+k-\delta_{2}+\delta_{1}\right)<1,
\end{aligned}
$$

then every solution $(x(t), y(t))$ of system (1) with $x(t)$ bounded is oscillatory.

Proof. Let $(x(t), y(t))$ be a nonoscillatory solution of system (1) with $x(t)$ bounded. Without loss of generality, we may assume that $x(t)$ is eventually positive and bounded for all $t \geq t_{1} \geq t_{0}$. From the second equation of system (1), we obtain $y^{\Delta}(t) \leq 0$ for sufficiently large $t \geq t_{1}$. In view of Lemma 1 , we have two cases for sufficiently large $t_{2} \geq t_{1}$ :
(a) $y(t)<0$ for $t \geq t_{2}$;
(b) $y(t)>0$ for $t \geq t_{2}$.

Case (a). Because $y(t)$ is negative and nonincreasing, there is a constant $L>0$ such that

$$
y(t) \leq-L, \quad t \geq t_{2} .
$$

Since $x(t)$ and $a(t)$ are bounded, $z(t)$ defined by (15) is bounded. Integrating the first equation of system (1) from $t_{2}$ to $t$ and using (24), we have

$$
\begin{aligned}
z(t)-z\left(t_{2}\right) & =\int_{t_{2}}^{t} p(s) f_{1}(y(s)) \Delta s \\
& \leq f_{1}(-L) \int_{t_{2}}^{t} p(s) \Delta s, \quad t \geq t_{2} .
\end{aligned}
$$

From (25), we get $\lim _{t \rightarrow \infty} z(t)=-\infty$, which contradicts the fact that $z(t)$ is bounded. Case (a) cannot occur.

Case (b). We consider two possibilities.

(i) Let $z(t)>0$ for $t \geq t_{2}$ be sufficiently large. Because $z(t)$ is nondecreasing, there is a positive constant $M$ such that

$$
z(t) \geq M
$$

for all sufficiently large $t \geq t_{2}$. From (15) and the hypothesis $\left(\mathrm{H}_{4}\right)$, we obtain

$$
q(t) z\left(\tau_{2}(t)\right) \leq q(t) x\left(\tau_{2}(t)\right) \leq q(t) \frac{f_{2}\left(x\left(\tau_{2}(t)\right)\right)}{\beta_{2}},
$$

for all sufficiently large $t \geq t_{2}$. Integrating the second equation of system (1) from $t$ to $b$, using (27), and then letting $b \rightarrow \infty$, we get

$$
y(t) \geq \beta_{2} \int_{t}^{\infty} q(s) z\left(\tau_{2}(s)\right) \Delta s,
$$

for all sufficiently large $t \geq t_{2}$. From condition (22), we obtain

$$
\begin{aligned}
& \lim _{t \rightarrow \infty} \sup \left\{\int_{t}^{\infty} A(s) q(s) \Delta s\right\} \\
& \geq \lim _{t \rightarrow \infty} \sup \left\{A(t) \int_{t+\delta_{2}}^{\infty} q(s) \Delta s\right\}>\frac{1}{\beta_{1} \beta_{2}} .
\end{aligned}
$$

We claim that condition (22) implies

$$
\int_{\alpha}^{\infty} A(s) q(s) \Delta s=\infty, \quad \alpha \geq t_{0} .
$$


Otherwise, if $\int_{\alpha}^{\infty} A(s) q(s) \Delta s<\infty$, we can choose an integer $\gamma \geq \alpha$ so large that $\int_{\gamma}^{\infty} A(s) q(s) \Delta s<1 /\left(\beta_{1} \beta_{2}\right)$, which contradicts (29). From (9) and the monotonicity of $y(t)$, we have

$$
\begin{aligned}
\int_{\alpha}^{t} A(s) f_{1}^{\Delta}(y(s)) \Delta s \\
=A(t) f_{1}(y(t))-A(\alpha) f_{1}(y(\alpha)) \\
\quad-\int_{\alpha}^{t} A^{\Delta}(s) f_{1}(y(\sigma(s))) \Delta s \\
=A(t) f_{1}(y(t))-A(\alpha) f_{1}(y(\alpha)) \\
\quad-\int_{\alpha}^{t} p(s) f_{1}(y(\sigma(s))) \Delta s \\
\geq A(t) f_{1}(y(t))-A(\alpha) f_{1}(y(\alpha)) \\
\quad-\int_{\alpha}^{t} p(s) f_{1}(y(s)) \Delta s=A(t) f_{1}(y(t)) \\
\quad-A(\alpha) f_{1}(y(\alpha))-z(t)+z(\alpha) .
\end{aligned}
$$

From (26), (27), (31), and the second equation of system (1), we have

$$
\begin{aligned}
& \int_{\alpha}^{t} A(s) f_{1}^{\Delta}(y(s)) \Delta s \\
& \quad \leq K \int_{\alpha}^{t} A(s) y^{\Delta}(s) \Delta s \\
& \quad=K \int_{\alpha}^{t} A(s)\left[-q(s) f_{2}\left(x\left(\tau_{2}(s)\right)\right)\right] \Delta s \\
& \quad \leq K \beta_{2} \int_{\alpha}^{t} A(s)\left[-q(s) z\left(\tau_{2}(s)\right)\right] \Delta s \\
& \leq-M K \beta_{2} \int_{\alpha}^{t} A(s) q(s) \Delta s, \\
& M K \beta_{2} \int_{\alpha}^{t} A(s) q(s) \Delta s \leq-A(t) f_{1}(y(t))+A(\alpha) f_{1}(y(\alpha)) \\
& \quad+z(t)-z(\alpha), \quad t \geq \alpha .
\end{aligned}
$$

Combining the last inequality with (30), we have

$$
\begin{gathered}
\lim _{t \rightarrow \infty}\left[z(t)-A(t) f_{1}(y(t))\right]=\infty, \\
z(t) \geq A(t) f_{1}(y(t)) \geq \beta_{1} A(t) y(t),
\end{gathered}
$$

for all sufficiently large $t \geq t_{2}$. The last inequality together with (28) and the monotonicity of $z(t)$ implies

$$
\begin{aligned}
z(t) & \geq \beta_{1} \beta_{2} A(t) \int_{t}^{\infty} q(s) z\left(\tau_{2}(s)\right) \Delta s \\
& \geq \beta_{1} \beta_{2} A(t) \int_{t+\delta_{2}}^{\infty} q(s) z\left(\tau_{2}(s)\right) \Delta s \\
& \geq \beta_{1} \beta_{2} A(t) z(t) \int_{t+\delta_{2}}^{\infty} q(s) \Delta s,
\end{aligned}
$$

and $1 \geq \beta_{1} \beta_{2} A(t) \int_{t+\delta_{2}}^{\infty} q(s) \Delta s$, for all sufficiently large $t \geq t_{2}$, which contradicts (22). This case cannot occur.

(ii) Let $z(t)<0$ for all sufficiently large $t \geq t_{2}$. From (15), we have

$$
z\left(t-\delta_{2}+\delta_{1}\right)>-a\left(t-\delta_{2}+\delta_{1}\right) x\left(t-\delta_{2}\right),
$$

where $t$ is sufficiently large and

$$
\frac{-\beta_{2} q(t) z\left(t-\delta_{2}+\delta_{1}\right)}{a\left(t-\delta_{2}+\delta_{1}\right)} \leq \beta_{2} q(t) \times\left(\tau_{2}(t)\right) .
$$

In view of the hypothesis and the second equation of system (1), the last inequality implies

$$
y^{\Delta}-\frac{\beta_{2} q(t) z\left(t-\delta_{2}+\delta_{1}\right)}{a\left(t-\delta_{2}+\delta_{1}\right)} \leq 0, \quad t \geq t_{2} .
$$

Integrating (37) from $t$ to $t+k$, we have

$$
y(t)+\int_{t}^{t+k} \frac{\beta_{2} q(s) z\left(s-\delta_{2}+\delta_{1}\right)}{a\left(s-\delta_{2}+\delta_{1}\right)} \Delta s \geq 0 .
$$

Multiplying the last inequality by $\beta_{1} p(t)$ and then using the monotonicity of $z(t)$ and the first equation of system (1), we have

$$
\begin{aligned}
& z^{\Delta}(t)+\beta_{1} \beta_{2} p(t) z\left(t+k-\delta_{2}+\delta_{1}\right) \\
& \quad \times \int_{t}^{t+k} \frac{q(s)}{a\left(s-\delta_{2}+\delta_{1}\right)} \Delta s \geq 0, \quad t \geq t_{6} .
\end{aligned}
$$

By condition (23) and Lemma 3, the last inequality cannot have an eventually negative solution. This contradicts the assumption that $z(t)<0$ eventually. The proof is complete.

Theorem 5. Let (16) hold. Assume that $0<a(t) \leq 1, \tau_{i}=$ $t-\delta_{i}$, and there exists an constant $k$ such that $\delta_{2}>k+\delta_{1}$ and conditions (22) and (23) are satisfied. Then, all solutions of system (1) are oscillatory.

Proof. Let $(x(t), y(t))$ be a nonoscillatory solution of system (1). Without loss of generality, we may assume that $x(t)$ is positive for $t \geq t_{1}$. As in the proof of Theorem 4, we have two cases.

Case (a). Analogous to the proof of case (a) of Theorem 4, we can show that $\lim _{t \rightarrow \infty} z(t)=-\infty$. By Lemma 2, 
$x(t)$ is bounded, and, hence, $z(t)$ is bounded, which is a contradiction. Hence, case (a) cannot occur.

Case (b). The proof of this case is similar to that of Theorem 4, and, hence, the details are omitted. The proof is now complete.

Remark 6. Theorems 4-5 include Theorems 5-6 in [4].

\section{The Case $-1<a(t) \leq 0$}

In this section, we always assume that

$$
-1<-c \leq a(t) \leq 0,
$$

where $c$ is a positive constant.

Lemma 7. Suppose that $\left(\mathrm{H}_{2}\right)-\left(\mathrm{H}_{4}\right)$ and (40) hold, and $(x(t), y(t))$ is a nonoscillatory solution of system (1). Then, $x(t) y(t)$ is eventually positive.

Proof. Without loss of generality, we may assume that $x(t)>$ $0, t \geq t_{0}$. Then, in view of $\left(H_{3}\right)$ and the hypothesis on $f_{2}$, we have $y^{\Delta}(t) \leq 0$ for all $t \geq t_{1} \geq t_{0}$ from the second equation of system (1). We claim that

$$
y(t)>0, \quad t \geq t_{1} .
$$

Otherwise, there exists $t_{2} \geq t_{1}$ such that

$$
y(t)<0, \quad t \geq t_{2} \text {. }
$$

Now, from the first equation of system (1) and the monotonicity of $y(t)$, we have

$$
z^{\Delta}(t) \leq 0
$$

Integrating the first equation of system (1) from $t_{2}$ to $t$, we get

$$
\begin{aligned}
z(t)-z\left(t_{2}\right) & =\int_{t_{2}}^{t} p(s) f_{1}(y(s)) \Delta s \\
& \leq \beta_{1} \int_{t_{2}}^{t} p(s) y(s) \Delta s \\
& \leq \beta_{1} y\left(t_{2}\right) \int_{t_{2}}^{t} p(s) \Delta s .
\end{aligned}
$$

From $\left(H_{3}\right),(42)$, and the last inequality, we obtain $\lim _{t \rightarrow \infty} z(t)=-\infty$. But in view of (15), we have $z(t) \geq x(t)$. So, $\lim _{t \rightarrow \infty} x(t)=-\infty$. This contradicts $x(t)>0$. This completes the proof of the lemma.

Theorem 8. Suppose that $\left(H_{2}\right)-\left(H_{4}\right)$ and (40) hold, and $f_{1} \in$ $C^{1}(\mathbb{R}, \mathbb{R})$ with $f_{1}^{\prime}(u) \geq K>0$. If

$$
\lim _{t \rightarrow \infty} \sup \left\{A(t) \int_{t+\delta_{2}}^{\infty} q(s) \Delta s\right\}>\frac{1}{\beta_{1} \beta_{2}(1-c)} .
$$

Then, every solution $(x(t), y(t))$ of system (1) is oscillatory.
Proof. Suppose that $(x(t), y(t))$ is a nonoscillatory solution of system (1). From Lemma 7, without loss of generality, we may assume that

$$
x(t)>0, \quad y(t)>0, \quad t \geq t_{1} .
$$

Combining (15) with (40), we obtain that $z(t)>0$ for $t \geq t_{1}$. From the first equation of system (1), we get $z^{\Delta}(t) \geq 0$ for all $t \geq t_{1}$. So, $z(t)$ is nondecreasing. There is a positive constant $M$ such that

$$
z(t) \geq M, \quad t \geq t_{2} \geq t_{1} .
$$

From (15), we get

$$
\begin{aligned}
x(t) & =z(t)+a(t) x\left(\tau_{1}(t)\right) \\
& =z(t)+a(t)\left[z\left(\tau_{1}(t)\right)+a\left(\tau_{1}(t)\right) x\left(\tau_{1}\left(\tau_{1}(t)\right)\right)\right] \\
& =z(t)+a(t) z\left(\tau_{1}(t)\right)+a(t) a\left(\tau_{1}(t)\right) x\left(\tau_{1}\left(\tau_{1}(t)\right)\right) \\
& >z(t)+a(t) z\left(\tau_{1}(t)\right) \\
& \geq z(t)+a(t) z(t) \\
& =(1+a(t)) z(t)
\end{aligned}
$$

for $t \geq t_{2}$. The last inequality together with $\left(H_{4}\right)$ implies

$$
q(t) z\left(\tau_{2}(t)\right) \leq \frac{q(t) x\left(\tau_{2}(t)\right)}{1+a\left(\tau_{2}(t)\right)} \leq \frac{q(t) f_{2}\left(x\left(\tau_{2}(t)\right)\right)}{\left[1+a\left(\tau_{2}(t)\right)\right] \beta_{2}}
$$

Integrating the second equation of system (1) from $t$ to $b$, using (49), and then letting $b \rightarrow \infty$, we obtain

$$
y(t) \geq(1-c) \beta_{2} \int_{t}^{\infty} q(s) z\left(\tau_{2}(s)\right) \Delta s
$$

From condition (45), we have

$$
\begin{aligned}
\frac{1}{(1-c) \beta_{1} \beta_{2}} & <\lim _{t \rightarrow \infty} \sup \left\{A(t) \int_{t+\delta_{2}}^{\infty} q(s) \Delta s\right\} \\
& \leq \lim _{t \rightarrow \infty} \sup \int_{t}^{\infty} A(s) q(s) \Delta s .
\end{aligned}
$$

We claim that condition (45) implies

$$
\int_{\alpha}^{\infty} A(s) q(s) \Delta s=\infty, \quad \alpha \geq t_{0} .
$$

In fact, if $\int_{\alpha}^{\infty} A(s) q(s) \Delta s<\infty$, we can choose a constant $\gamma \geq \alpha$ so large that $\int_{\gamma}^{\infty} A(s) q(s) \Delta s<1 /\left(\beta_{1} \beta_{2}(1-c)\right)$, which 
contradicts (51). From (9) and the monotonicity of $y(t)$, we have

$$
\begin{aligned}
\int_{\alpha}^{t} A(s) f_{1}^{\Delta}(y(s)) \Delta s= & A(t) f_{1}(y(t))-A(\alpha) f_{1}(y(\alpha)) \\
& -\int_{\alpha}^{t} A^{\Delta}(s) f_{1}(y(\sigma(s))) \Delta s \\
= & A(t) f_{1}(y(t))-A(\alpha) f_{1}(y(\alpha)) \\
& -\int_{\alpha}^{t} p(s) f_{1}(y(\sigma(s))) \Delta s \\
\geq & A(t) f_{1}(y(t))-A(\alpha) f_{1}(y(\alpha)) \\
& -\int_{\alpha}^{t} p(s) f_{1}(y(s)) \Delta s \\
= & A(t) f_{1}(y(t))-A(\alpha) f_{1}(y(\alpha)) \\
& -z(t)+z(\alpha) .
\end{aligned}
$$

From (49) and (53) and the second equation of system (1), we have

$$
\begin{aligned}
& \int_{\alpha}^{t} A(s) f_{1}^{\Delta}(y(s)) \Delta s \leq K \int_{\alpha}^{t} A(s) y^{\Delta}(s) \Delta s \\
&= K \int_{\alpha}^{t} A(s)\left[-q(s) f_{2}\left(x\left(\tau_{2}(s)\right)\right)\right] \Delta s \\
& \leq K \beta_{2} \int_{\alpha}^{t} A(s)\left[1+a\left(\tau_{2}(s)\right)\right] \\
& \times\left[-q(s) z\left(\tau_{2}(s)\right)\right] \Delta s \\
& \leq-M K \beta_{2}(1-c) \int_{\alpha}^{t} A(s) q(s) \Delta s, \\
& M K \beta_{2}(1-c) \int_{\alpha}^{t} A(s) q(s) \Delta s \\
& \leq-A(t) f_{1}(y(t))+A(\alpha) f_{1}(y(\alpha)) \\
& \quad+z(t)-z(\alpha), \quad t \geq \alpha .
\end{aligned}
$$

Combining the last inequality with (52), we have

$$
\begin{gathered}
\lim _{t \rightarrow \infty}\left[z(t)-A(t) f_{1}(y(t))\right]=\infty, \\
z(t) \geq A(t) f_{1}(y(t)) \geq \beta_{1} A(t) y(t), \quad t \geq t_{3} \geq t_{2} .
\end{gathered}
$$

The last inequality together with (28) and the monotonicity of $z(t)$ implies

$$
\begin{aligned}
z(t) & \geq \beta_{1} \beta_{2}(1-c) A(t) \int_{t}^{\infty} q(s) z\left(\tau_{2}(s)\right) \Delta s \\
& \geq \beta_{1} \beta_{2} A(t)(1-c) \int_{t+\delta_{2}}^{\infty} q(s) z\left(\tau_{2}(s)\right) \Delta s \\
& \geq \beta_{1} \beta_{2}(1-c) A(t) z(t) \int_{t+\delta_{2}}^{\infty} q(s) \Delta s
\end{aligned}
$$

and $1 \geq \beta_{1} \beta_{2}(1-c) A(t) \int_{t+\delta_{2}}^{\infty} q(s) \Delta s, t \geq t_{3}$, which contradicts (45). This case cannot occur. The proof is complete.

Remark 9. Theorem 8 improves Theorem 3.1 in [13], because condition (45) is weaker than condition (5) assumed in [13].

\section{Some Examples}

In this section, we present examples to illustrate the results obtained in the previous sections.

Example 10. Consider the system

$$
\begin{aligned}
& {\left[x(t)-\frac{1}{4} x(t-b)\right]^{\Delta}=t(t+2 b) y(t),} \\
& y^{\Delta}(t)=-\frac{c}{t(t+b)} x(t-4 b)
\end{aligned}
$$

where $\mathbb{T}=b \mathbb{N}=\{b n \mid n \in \mathbb{N}\}$ and $b, c$ is a positive constant. Here, $f(u)=g(u)=u, a(t)=1 / 4, \delta_{1}=b, \delta_{2}=4 b, p(t)=$ $t(t+2 b), q(t)=c /(t(t+b))$. Choose $k=1$, since

$$
\begin{aligned}
A(t) & =\int_{0}^{t} p(s) \Delta s=b^{3} \sum_{i=0}^{n} i(i+2) \\
& =b^{3} \sum_{i=1}^{n+1}(i+1)(i-1)=b^{3} \frac{2 n^{3}+9 n^{2}+12 n}{6}
\end{aligned}
$$

then, conditions (22) and (23) are

$$
\begin{aligned}
& \lim _{t \rightarrow \infty} \sup \left\{A(t) \int_{t+\delta_{2}}^{\infty} q(s) \Delta s\right\} \\
& =\lim _{n \rightarrow \infty} \sup \left\{b^{3} \frac{2 n^{3}+9 n^{2}+12 n}{6} \frac{1}{b} \sum_{s=n+4}^{\infty} \frac{c}{s(s+1)}\right\}=\infty, \\
& \lim _{n \rightarrow \infty} \inf \left\{b^{3} \sum_{s=n-2}^{n-1} s(s+2)\left[\frac{1}{b} \sum_{t=s}^{s+1} \frac{4 c}{t(t+1)}\right]\right\}=16 b^{2} c .
\end{aligned}
$$

For $16 b^{2} c>1$, all the conditions of Theorem 4 are satisfied, and so all solutions of the system (57) are oscillatory. But the results [4] are not applicable.

Example 11. Consider the system

$$
\begin{aligned}
& {\left[x(t)+a(t) x\left(\tau_{1}(t)\right)\right]^{\Delta}=t f_{1}(y(t)),} \\
& y^{\Delta}(t)=-\frac{1}{t^{3 / 2}} f_{2}\left(x\left(\tau_{2}(t)\right)\right),
\end{aligned}
$$

where $\mathbb{T}=\mathbb{R}$ and $-1<a(t) \leq 0$. Here, $p(t)=t, q(t)=1 / t^{3 / 2}$. Since

$$
A(t)=\int_{0}^{t} p(s) \Delta s=\int_{0}^{t} s d s=\frac{1}{2} t^{2}
$$


then condition $(45)$ is

$$
\begin{aligned}
& \lim _{t \rightarrow \infty} \sup \left\{A(t) \int_{t+\delta_{2}}^{\infty} q(s) \Delta s\right\} \\
& =\lim _{t \rightarrow \infty} \sup \left\{\frac{1}{2} t^{2} \int_{t+\delta_{2}}^{\infty} \frac{1}{s^{3 / 2}} d s\right\}=\infty .
\end{aligned}
$$

Condition (45) is satisfied. Hence, by Theorem 8, all solutions of system (60) are oscillatory.

\section{Acknowledgment}

This work is supported by the NSF of Shandong, China (BS2011DXD11).

\section{References}

[1] S. Hilger, "Analysis on measure chains-a unified approach to continuous and discrete calculus," Results in Mathematics, vol. 18, no. 1-2, pp. 18-56, 1990.

[2] R. Agarwal, M. Bohner, D. O'Regan, and A. Peterson, "Dynamic equations on time scales: a survey," Journal of Computational and Applied Mathematics, vol. 141, no. 1-2, pp. 1-26, 2002.

[3] M. Bohner and A. Peterson, Dynamic Equations on Time Scales. An Introduction with Applications, Birkhäuser, Boston, Mass, USA, 2001.

[4] E. Thandapani and P. Mohan Kumar, "Oscillation of difference systems of the neutral type," Computers \& Mathematics with Applications, vol. 54, no. 4, pp. 556-566, 2007.

[5] L. Hanuštiaková and R. Olach, "Nonoscillatory bounded solutions of neutral differential systems," Nonlinear Analysis: Theory, Methods \& Applications, vol. 68, no. 7, pp. 1816-1824, 2008.

[6] L. Erbe, A. Peterson, and S. H. Saker, "Oscillation criteria for second-order nonlinear delay dynamic equations," Journal of Mathematical Analysis and Applications, vol. 333, no. 1, pp. 505522, 2007.

[7] S. H. Saker and D. O'Regan, “New oscillation criteria for second-order neutral functional dynamic equations via the generalized Riccati substitution," Communications in Nonlinear Science and Numerical Simulation, vol. 16, no. 1, pp. 423-434, 2011.

[8] D.-X. Chen, "Oscillation and asymptotic behavior for $n$ th-order nonlinear neutral delay dynamic equations on time scales," Acta Applicandae Mathematicae, vol. 109, no. 3, pp. 703-719, 2010.

[9] R. N. Rath, N. Misra, and L. N. Padhy, "Oscillatory and asymptotic behaviour of a nonlinear second order neutral differential equation," Mathematica Slovaca, vol. 57, no. 2, pp. 157-170, 2007.

[10] M. Bohner and A. Peterson, Eds., Advances in Dynamic Equations on Time Scales, Birkhäuser, Boston, Mass, USA, 2003.

[11] R. P. Agarwal and M. Bohner, "Basic calculus on time scales and some of its applications," Results in Mathematics, vol. 35, no. 1-2, pp. 3-22, 1999.

[12] B. G. Zhang and X. Deng, "Oscillation of delay differential equations on time scales," Mathematical and Computer Modelling, vol. 36, no. 11-13, pp. 1307-1318, 2002.

[13] S.-C. Fu and M.-L. Lin, "Oscillation and nonoscillation criteria for linear dynamic systems on time scales," Computers \& Mathematics with Applications, vol. 59, no. 8, pp. 2552-2565, 2010. 


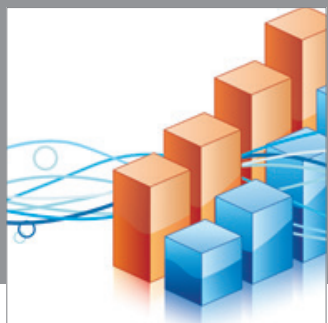

Advances in

Operations Research

mansans

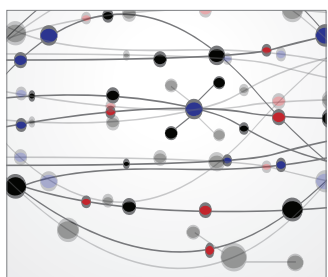

The Scientific World Journal
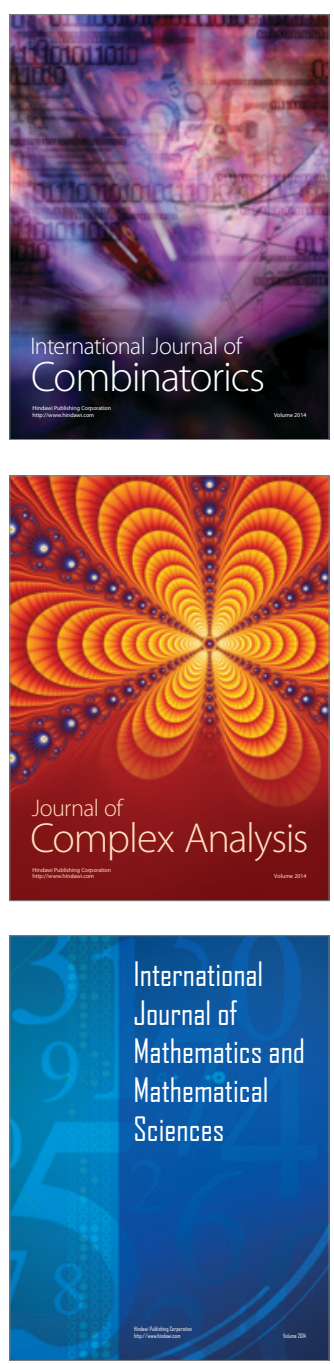
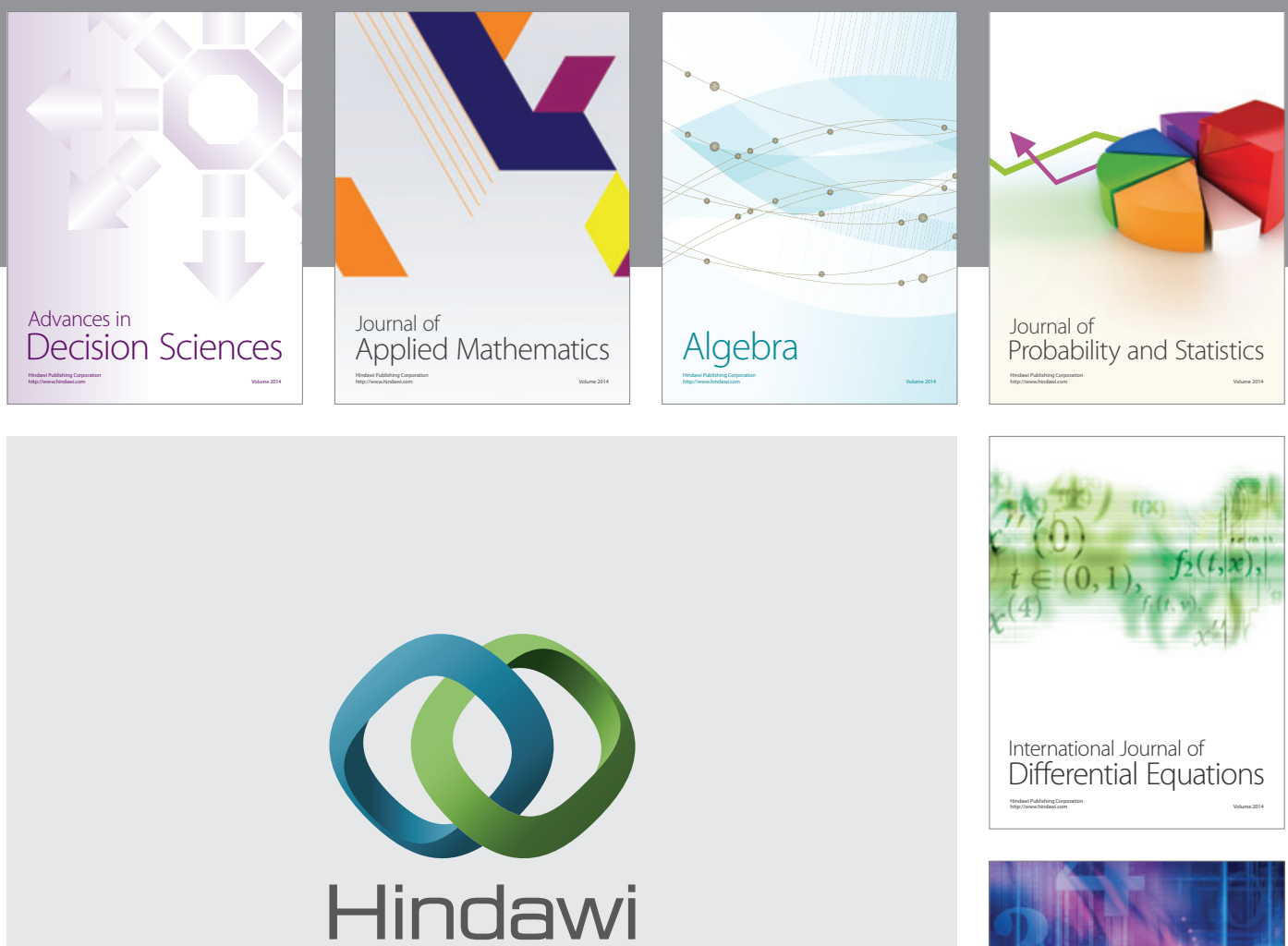

Submit your manuscripts at http://www.hindawi.com
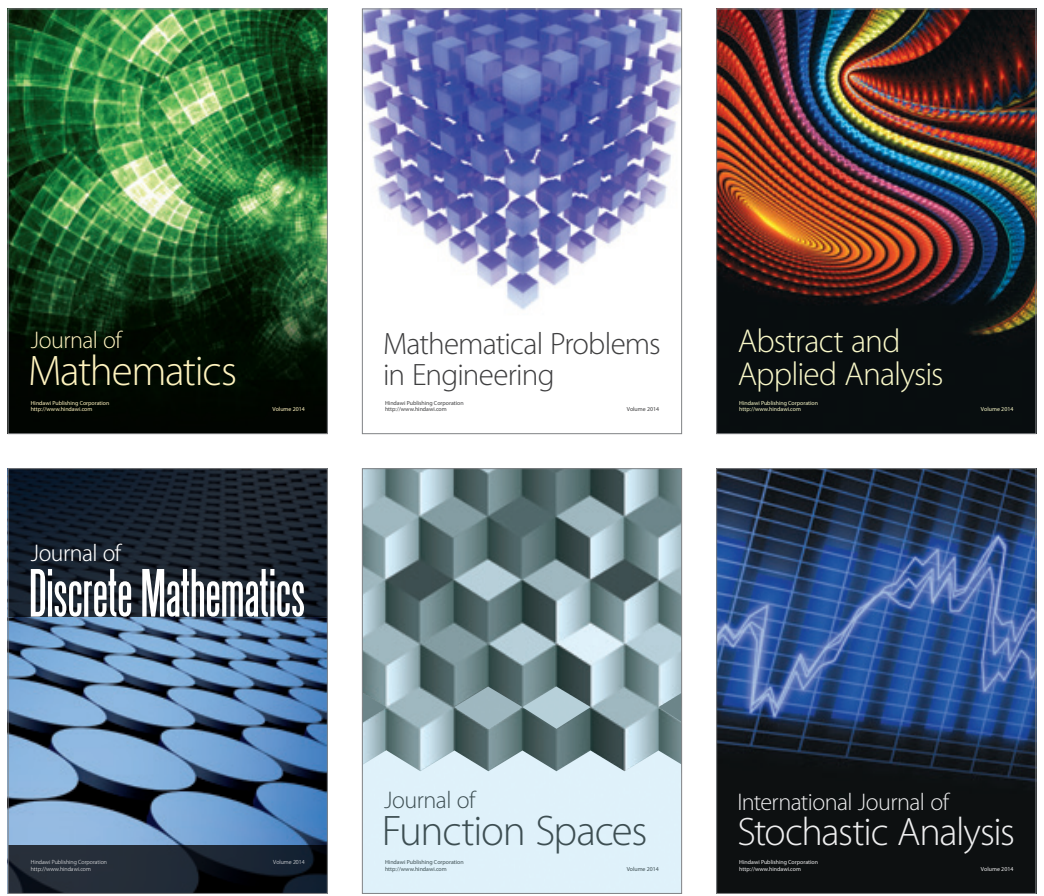

Journal of

Function Spaces

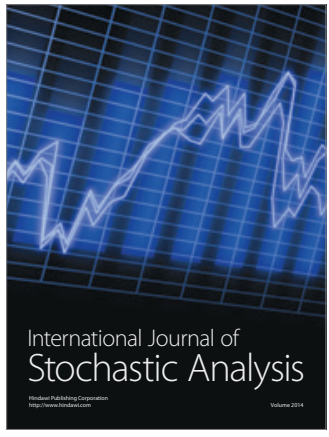

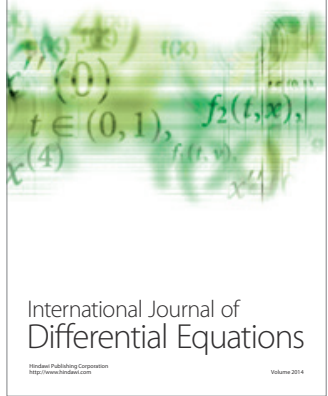
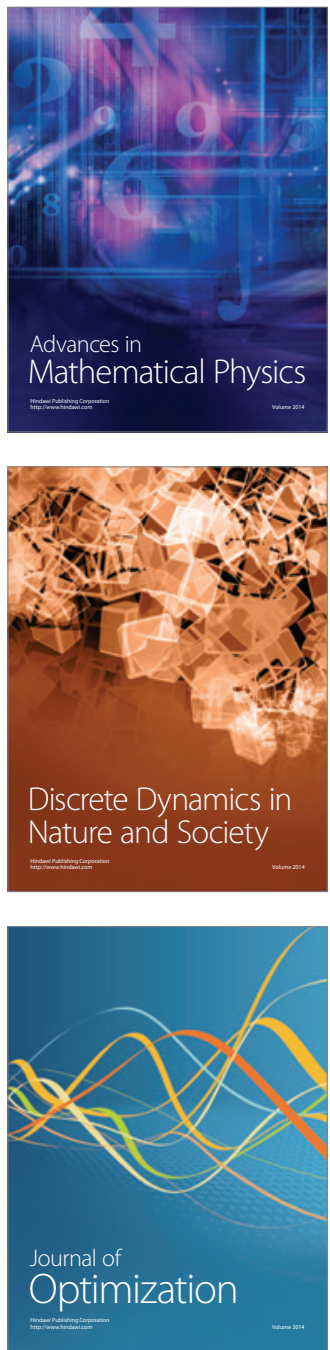